\title{
Multiplex Immunolabeling and Imaging of Functionally Essential Kidney Structures in X-CLARITY-Cleared Tissue
}

\author{
Pauline Mochama ${ }^{1}$, Roman Tyshynsky ${ }^{2}$ and Mark A. Sanders ${ }^{1 *}$ \\ 1. University Imaging Centers, University of Minnesota, Minneapolis, MN, USA. \\ 2. Department of Neuroscience, University of Minnesota, Minneapolis, MN, USA. \\ * Corresponding author: msanders@umn.edu
}

Tissue clearing techniques render tissues transparent and allow for 3D analysis of anatomical structure in intact volumes of tissues. CLARITY-based techniques embed tissues in a porous hydrogel matrix that preserves tissue architecture during clearing and facilitates effective immunostaining in thick tissue volumes [1]. Furthermore, hydrogel-embedded tissue is highly amenable to multiple rounds of immunostaining and imaging without significant tissue loss [2]. In this study, we utilize the XCLARITY tissue clearing system (Logos Biosystems) to clear kidney tissue sections of varying thickness and demonstrate techniques for antibody labeling, stripping, and reprobing of cleared tissue sections. This allows for the imaging and mapping of multiple structurally and functionally important components of the kidney in $3 \mathrm{D}$ within the same tissue.

$4 \%$ PFA-fixed sections $(100 \mu \mathrm{m}, 500 \mu \mathrm{m}$ and $1000 \mu \mathrm{m})$ were cleared as follows. Fixed tissues were rinsed several times with $1 \mathrm{X}$ phosphate-buffered saline (PBS) over 24 hours. Tissues were then immersed in XCLARITY hydrogel monomer plus initiator solution for $24 \mathrm{~h}$ at $4^{\circ} \mathrm{C}$ and then polymerized for $3 \mathrm{~h}$ at $37^{\circ} \mathrm{C}$ in a vacuum chamber $(-90 \mathrm{kPa})$. Following polymerization, tissues were de-lipidized in an electrophoretic tissue clearing (ETC) chamber with a current of 1.1A and a circulation speed of 50rpm at $37^{\circ} \mathrm{C}$ for $6 \mathrm{~h}$. Samples were washed with PBST (1X PBS, 0.1\% Triton X-100) with 5 changes over six hours to eliminate residual SDS from the clearing solution and then incubated in blocking buffer (SEA blocking buffer, Thermo Fisher) at $4^{\circ} \mathrm{C}$ overnight $(>14 \mathrm{~h})$. Sections were then incubated in primary antibodies (anti-nephrin and anti-CGRP) at a dilution of 1:500 for $24 \mathrm{~h}$ at $37^{\circ} \mathrm{C}$. Secondary antibodies, Alexa-488 and a Cy3-conjugate respectively, were prepared at a dilution of 1:500 and the tissues incubated in this solution for $24 \mathrm{~h}$ at $37^{\circ} \mathrm{C}$ with extensive rinsing with PBST between antibody incubations. Prior to imaging, samples were placed in X-CLARITY RIMS mounting solution overnight. Cleared, labeled tissues were imaged using the RS-G4 ribbon-scanning, upright confocal microscope (Caliber I.D.). Images were viewed and analyzed using Imaris, 9.2.1 software (Bitplane Oxford Instruments, Concord, MA)

For reprobing experiments in the same tissue set, ETC clearing solution (Logos Biosystems) was utilized for antibody stripping. Previously antibody-labeled kidney sections were placed in an ETC chamber (1.1A, 37C, 70rpm) for $2 \mathrm{~h}$. Delabeled sections were observed under the microscope to ensure complete elimination of labeling. The stripping process was repeated until no fluorescence from labeled structures was observed. Sections were then rinsed with PBST and incubated in a second primary antibody pair (anti-TH and anti-SMA) followed by incubation in Alexa-488 and Cy3-conjugated secondary antibodies, respectively. Kidney sections were mounted in RIMS once again and re-imaged as outlined above.

Results indicated that the kidney sections were efficiently cleared using the X-CLARITY technique (Fig 1) with no significant or remarkable changes in tissue dimensions, a noted advantage of this technique. 
Following antibody labeling, we observed the expected labeling patterns; however, in the 1-mm kidney sections, labeling was diminished in the mid-plane region of the tissue (Fig 2).

To improve antibody penetration and labeling deeper into the tissue, we tested a commercial immunostaining kit developed for improved labeling of cleared tissue samples known as DeepLabel Antibody Staining Kit (Logos Biosystems). We saw some improvement in antibody penetration and labeling with kidney slices treated and labeled with DeepLabel reagents and antibodies over kidney slices that were conventionally labeled. However, labeling was still absent in some mid-section areas of the 1-mm thick tissue sections. These results could be due to reagent access or infiltration issues or could indicate that the store of available unbound antibody may be depleted over time due to the abundance of epitopes. In current experiments we are testing the effects of replenishing available antibody by adding fresh aliquots or primary antibodies at multiple points during the incubation period.

We were able to successfully eliminate initial antibody labeling and relabel kidney sections with a second set of antibodies targeting new antigens. These results demonstrate the efficiency of electrophoretic clearing systems in not just tissue clearing but in antibody stripping as well. No labeling of the initially targeted glomeruli and enervation pathways were visible in the stripped and relabled tissue. Antibody stripping involves a delicate balance between effectively dislodging bound antibodies and maintaining the structural and epitope integrity of tissues. Electrophoretic clearing techniques when applied to hydrogel-embedded tissue appear to be effective in maintaining this balance.

\section{References:}

[1] J Phillips et al., Scientific Reports 6 (2016), p. 26013.

[2] MM Bolognesi et al., Journal of Histochemistry \& Cytochemistry 65 (2017), p. 431.

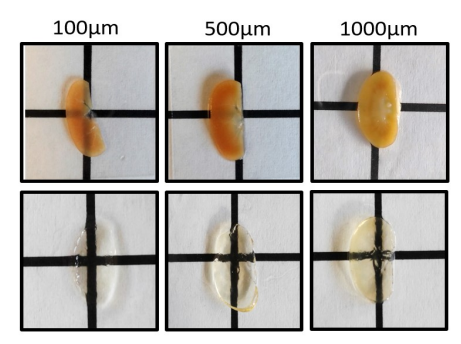

Figure 1. Kidney sections cleared using X-CLARITY.

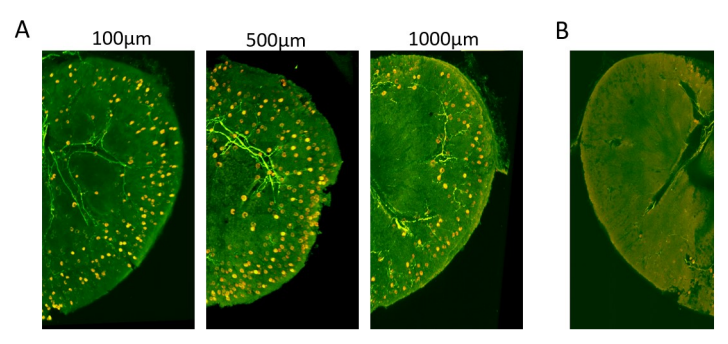

Figure 2. A) Immunofluorescent labeling of renal nerves and glomeruli in X-CLARITY cleared kidney tissue sections. B) Confocal z-section at $~ 500$ micron depth of 1-mm kidney slice. Note reduced labeling. 